\author{
W. Möller \\ M. Kohlhäufl \\ K. Häußinger \\ J. Heyder

\section{Pneumologische Diagnostik mit Modellaerosolen} \\ IV. Teil: Clearance von Aerosolpartikeln aus dem Alveolarbereich. \\ Grundlagen und erste klinische Forschungsergebnisse
}

\author{
Pneumological Diagnostics by Means of Models Aerosols
}

\section{Zusammenfassung}

Die Elimination deponierter Partikel (anorganische und organische Partikeln, Bakterien, Viren) aus der Lungenperipherie (alveolare Clearance) erfolgt durch Phagozytose, Alveolarmakrophagen, intrazellulären Abbau (Digestion), Migration und Translokation. Die alveolare Clearance schwer löslicher Partikeln verläuft sehr langsam und ist primär an die Funktion der Alveolarmakrophagen geknüpft. Der Transport der Partikel zum Bronchialsystem ist beim Menschen von untergeordneter Bedeutung, so dass die Elimination der Partikeln vornehmlich durch Digestion (Lösung) innerhalb der Makrophagen bestimmt wird. Wenn keine Metabolisierung des gelösten Materials im Körper stattfindet, erfolgt die Ausscheidung aus dem Körper über den Urin. Die Pathophysiologie der alveolaren Clearance kann mittels Magnetopneumographie über einen langen Zeitraum untersucht werden. Dazu werden ferromagnetische Magnetit-Testpartikel durch kontrollierte Inhalation in der Lungenperipherie abgelagert. Nach der Magnetisierung und Ausrichtung in einem starken externen Magnetfeldpuls kann die Menge der retinierten Partikel mit einem empfindlichen supraleitenden Magnetfeldsensor (SQUID, superconducting quantum interference device) erfasst werden. Lange andauernder Zigarettenrauchkonsum und chronische Entzündungen in der Lungenperipherie (Sarkoidose, interstitielle Lungenfibrose) führen zu einer signifikanten Verminderung der alveolaren Clearance. Patienten mit chronischer Bronchitis zeigen hingegen nur eine geringe Beeinträchtigung der alveolaren Clearance.

\section{Abstract}

The elimination of deposited particles (inorganic and organic particles, bacteria, viruses) from the periphery of the human lung (alveolar clearance) implies phagocytosis by alveolar macrophages, intracellular digestion, migration and translocation. Alveolar clearance of poorly soluble particles happens very slowly and primarily depends on the function of alveolar macrophages. In humans, the transport of particles to the bronchial tree is of secondary relevance, suggesting that the elimination of particles primarily depends on digestion (dissolution) processes within macrophages. The dissolved material is excreted via urine, if there is no further metabolization within the body. The pathophysiology of the alveolar clearance mechanisms in the human lung can be studied by a magnetic tracer technique (magnetopneumography). Ferromagnetic magnetite test-particles are deposited in the periphery of the lung by controlled inhalation. After magnetization and particle alignment in a strong external magnetic field pulse, the amount of retained particles can be detected by a sensitive magnetic field sensor (SQUID, superconducting quantum interference device). Long lasting cigarette smoking and chronic lung inflammations (sarcoidosis, interstitial lung fibrosis) induce a significant impairment of alveolar clearance capacity, while patients with chronic bronchitis show only a moderate impairment of alveolar clearance. 


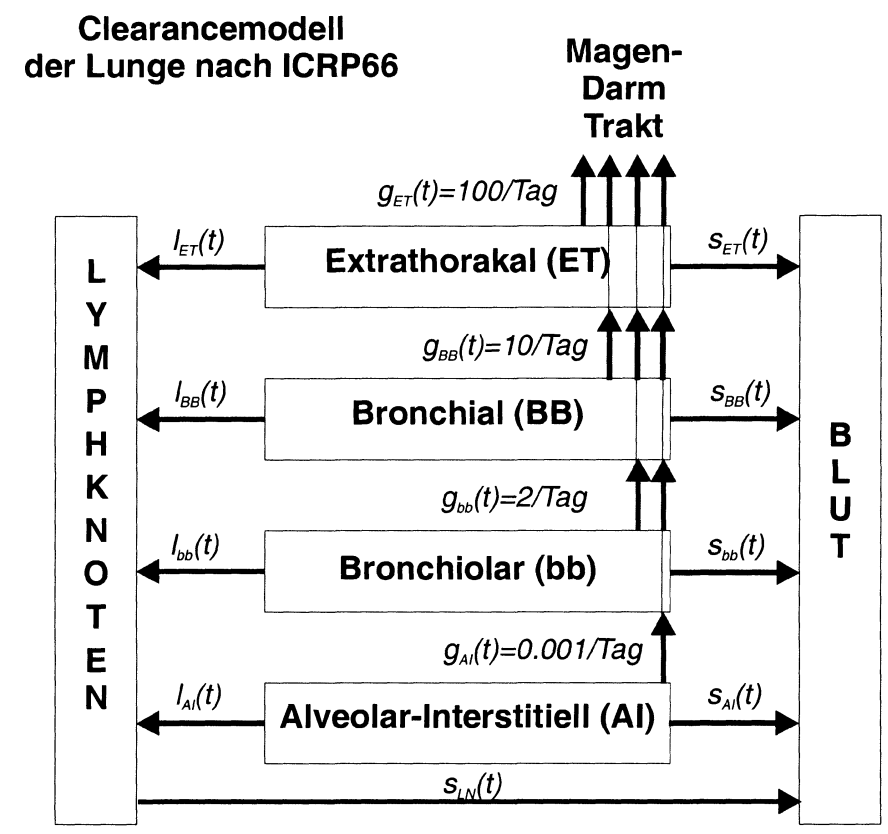

Abb. 1 Schematisches Modell der Clearance von Partikeln aus der Lunge des Menschen nach dem Modell der ICRP66 [16]. Die Clearanceraten aus den jeweiligen Kompartimenten i der Lunge werden beschrieben durch: $s_{i}(t)$ : Absorption ins Blut, $g_{i}(t)$ : mechanischer Transport zum Kehlkopf und Gastrointestinaltrakt, sowie $l_{i}(t)$ : mechanischer Transport zu den Lymphknoten.

\section{Einleitung}

Die Inhalation und Deposition von Modellaerosolen kann dazu verwendet werden, diagnostische Informationen über die Struktur und Funktion der menschlichen Lunge zu gewinnen. Der Einsatz von Modellaerosolen zur Untersuchung von peripheren Lungenstrukturveränderungen [18], zur Untersuchung des konvektiven Gastransports [19] und zur Untersuchung der Mukoziliarclearance [29] wurden bereits in dieser Zeitschrift beschrieben. Im vorliegenden 4. Teil soll die Partikelclearance aus der Lungenperipherie behandelt werden mit einem Überblick über die zugrunde liegenden Mechanismen und den augenblicklichen Stand der Forschung. Außerdem wird eine neue nichtinvasive magnetische Tracermethode (Magnetopneumographie, MPG) zur Untersuchung der peripheren Partikelclearance am Menschen mit ersten klinischen Forschungsergebnissen vorgestellt.

Die uns umgebende Atemluft enthält je nach Region (ländlich, städtisch) etwa 5000 bis 100000 Aerosolteilchen pro $\mathrm{cm}^{3}$ Luft. Damit gelangen mit jedem Atemzug einige Millionen Aerosolteilchen in die Lunge und werden hier teilweise abgelagert. In den verschiedenen anatomischen Regionen der Lunge herrschen unterschiedliche Abscheide- und Clearancemechanismen vor $[14,16]$. In den großen Atemwegen werden vor allem größere Teilchen (aerodynamischer Durchmesser $>5 \mu \mathrm{m}$ ) aufgrund der hohen Strömungsgeschwindigkeit und der Vielzahl der Verzweigungen durch Impaktionsprozesse abgeschieden. In den kleinen Atemwegen werden die Partikel durch Sedimentation abgeschieden. Somit wirken diese Regionen als Filter für den nachgeschalteten Alveolarbereich, in den vor allem Partikel mit einem aerodynamischen Durchmesser zwischen $1 \mu \mathrm{m}$ und $5 \mu \mathrm{m}$ vordringen können und durch Sedimentation abgelagert werden. Den unterschiedlichen anatomischen Regionen mit den jeweils unter- schiedlichen Depositionsmechanismen können auch spezifische Reinigungsmechanismen zugeordnet werden. Während die meisten der in den Atemwegen abgelagerten Partikel durch den mukoziliaren Schleimtransport innerhalb von 24 Stunden aus der Lunge heraustransportiert werden, verbleiben die in den Alveolen deponierten Partikel wesentlich länger in der Lunge und werden hauptsächlich durch Alveolarmakrophagen aus der Lunge entfernt.

Der makrophageninduzierten Reinigung können prinzipiell zwei verschiedene Mechanismen zugeordnet werden. Zunächst werden die abgelagerten Partikel innerhalb weniger Stunden von den Alveolarmakrophagen phagozytiert und in intrazellulare Vakuolen (Phagosomen) eingeschlossen. Damit wird prinzipiell eine permanente Sterilität der Lungenoberfläche gewährleistet. Ein Teil der mit Partikeln beladenen Makrophagen wandert zu den Atemwegen und kann dann die Lunge über den mukoziliaren Schleimtransport verlassen. Diese Fraktion der Reinigung wird auch als mechanische Clearancekomponente bezeichnet. Die restliche Fraktion verbleibt prinzipiell immer in den Makrophagen und muss durch chemische Lösung entfernt werden. Die Aufteilung zwischen mechanischem Transport in Richtung Atemwege und chemischer Lösung in den Makrophagen ist speziesabhängig [3]. Beim Menschen gelangen weniger als $1 / 3$ der in der Peripherie abgelagerten Partikel auf die mukoziliare „Rolltreppe“. Der überwiegende Teil verbleibt nach der Phagozytose in den Alveolarmakrophagen und muss hier abgebaut werden.

Die Untersuchung der Clearancemechanismen der Lunge wird im Allgemeinen mit radioaktiven Tracerpartikeln durchgeführt. Die Untersuchung der makrophageninduzierten Lungenclearance am Menschen gestaltet sich jedoch aus ethischen Gründen schwierig, da die Prozesse, im Vergleich etwa zur Mukoziliarclearance, sehr langsam ablaufen und Radionuklide mit langer Halbwertsdauer (ca. 6 Monate) eingesetzt werden müssen. Besonders für die Anwendung am Menschen bietet die alternative magnetische Tracertechnik (Magnetopneumographie, MPG) einen Ausweg aus dieser Problematik [10,35]. Dazu werden ferromagnetische Testpartikel inhaliert und deponiert. Die Partikel werden dann durch einen starken Magnetfeldpuls magnetisiert und parallel ausgerichtet, so dass ein makroskopisches remanentes Magnetfeld außerhalb des Thorax entsteht. Dies kann dann mit Hilfe von sensitiven supraleitenden Magnetfeldsensoren (SQUID's, Superconducting QUantum Interference Devices) erfasst werden. Diese Sensoren sind hochsensitiv und erlauben zusammen mit einer effektiven Abschirmung gegenüber äußeren Störfeldern allgemein den Nachweis weiterer biomagnetischer Felder des menschlichen Organismus (Magnetokardiogramm, [MKG] bzw. Magnetoenzephalogramm [MEG]).

In der vorliegenden Arbeit werden zunächst die grundlegenden Clearancemechanismen der Lunge des Menschen dargestellt und anhand des aktuellen ICRP-Modells erläutert. Unter Verwendung der biomagnetischen Nachweistechnik Magnetopneumographie werden Ergebnisse einer klinischen Studie zur alveolaren Langzeitreinigung von magnetischen Eisenoxidpartikeln bei Gesunden und bei Patienten mit Lungenkrankheiten vorgestellt und diskutiert. 
Clearance von Partikeln nach dem Modell

der ICRP-Publikation 66

Die zugrunde liegenden Mechanismen der Clearance von Partikeln aus der Lunge des Menschen sind in der Publikation 66 der International Commission on Radiological Protection (ICRP) zusammengefasst mit einer Übersicht über die verfügbare Literatur [16]. Aus diesen Arbeiten ist ein Modell über die Deposition und Clearance von inhalierten Aerosolteilchen erarbeitet worden, das in diesem Abschnitt kurz dargestellt werden soll. Dabei soll insbesondere die nach Inhalation von radioaktiven Aerosolen in der Lunge sowie in anderen Organen applizierte Dosis abgeschätzt werden. Die Clearancekinetik wird als Überlagerung fraktioneller Clearanceraten beschrieben:

$$
\lambda_{i}=-\frac{d R_{i}(t) / d t}{R_{i}(t)},
$$

wobei $\lambda_{i}$ die Gesamtclearancerate aus dem Kompartiment i der Lunge ist und $R_{i}(t)$ die Menge an Material in dieser Region beschreibt. Die Lunge wird dabei in insgesamt vier Kompartimente eingeteilt (Abb.1): extrathorakal (ET) mit Mund, Nase und Kehlkopf, bronchial (BB) mit den Bronchien, inklusive Trachea (Generation 0) bis zur 8. Generation, bronchiolar (bb) mit den Bronchiolen und den terminalen Bronchiolen (Generationen 9-15), sowie der alveolar-interstitiellen Region (AI) mit den respiratorischen Bronchiolen und den Alveolen (Generationen 16-23). Die Generationen ergeben sich als Anzahl der sequenziellen Verzweigungen des bronchialen Röhrensystems der Lunge aufgrund des anatomischen Modells von Weibel [40]. Als Clearancemechanismen werden 3 grundlegende Pfade angenommen:

- Absorption ins Blut,

- mechanischer Transport in den Magen-Darm-Trakt,

- mechanischer Transport in die Lymphknoten.

Aus allen vier Regionen gelangt Material auf diese Clearancepfade. Die Raten, mit denen das Material zu den Clearancepfaden transportiert wird, ist unabhängig von

- dem Ort der Partikelreposition im Atemtrakt,

- der physikalisch-chemischen Form des Materials und

- der Zeit seit der Ablagerung der Partikel im Atemtrakt.

Insgesamt ist die Clearance ein Zusammenspiel von mechanischem Transport und Absorption ins Blut. Das Modell geht weiterhin von den vereinfachenden Annahmen aus, dass

- die Clearanceraten von mechanischem Transport und der Absorption ins Blut unabhängig voneinander sind,

- die mechanischen Transportraten für alle Materialien gleich sind, und dass

- die Absorptionsrate materialabhängig ist, aber gleich in allen Regionen der Lunge.

Mit diesen Annahmen lassen sich die Clearanceraten im jeweiligen Kompartiment als Summe der Raten der jeweiligen Mechanismen beschreiben:

$$
\lambda_{i}(t)=m_{i}(t)+s_{i}(t), m_{i}(t)=g_{i}(t)+l_{i}(t),
$$

$m_{i}(t)$ beschreibt die Rate des mechanischen Transports, $s_{i}(t)$ die Rate der Absorption. Der mechanische Transport führt einerseits zum Gastrointestinaltrakt ( Rate $_{i}(t)$ ) sowie zu den Lymphknoten (Rate $l_{i}(t)$ ).

\section{Mukoziliare Clearance aus den Atemwegen}

Die Untersuchungen der mukoziliaren Clearance werden üblicherweise mit radioaktiv markierten Testaerosolen durchgeführt $[5,34,38]$. Hier hat sich vor allen das in der Nuklearmedizin häufig eingesetzte Radionuklid ${ }^{99 \mathrm{~m} T c}$ bewährt. ${ }^{99 \mathrm{~m} T c}$ hat eine Halbwertszeit von 6,3 Stunden und ist als reiner Gammastrahler mit einer Energie von etwa $130 \mathrm{keV}$ sehr einfach mit den in der Nuklearmedizin zur Verfügung stehenden bildgebenden Verfahren (Gammakamera) nachzuweisen. In wissenschaftlichen Studien am Menschen wurden auch ${ }^{111}$ In (Halbwertszeit 2,3 Tage) sowie ${ }^{198} \mathrm{Au}$ (Halbwertszeit 2,6 Tage) als Radiotracer verwendet. Mit abgeschirmten Szintillationsdetektoren kann die Strahlenbelastung der Probanden weit unterhalb der natürlichen Strahlenbelastung gehalten werden $[9,32,33]$.

Die Mechanismen der mukoziliaren Reinigung in den Atemwegen wurden eingehend in einem vorausgegangenen Artikel dieser Serie dargelegt [29], deshalb soll hier der Vollständigkeit halber nur kurz darauf eingegangen werden. Der mukoziliäre Schleimtransport ist sehr effektiv und entfernt die meisten Partikel innerhalb von 24 Stunden aus der Lunge. Bei Rauchern und bei Patienten mit chronischen Atemwegsentzündungen (Bronchitis, COPD) kommt es zu einer Verlangsamung der mukoziliaren Clearance, vor allem während der ersten 5-10 Stunden nach der Partikelablagerung, wobei sich allerdings nach 24 Stunden nur geringfügige Unterschiede zu lungengesunden Nichtrauchern ergeben. Bei Patienten mit einer Funktionsstörung der Zilien (Primäre Ciliäre Dyskinesie, PCD) ist der Mukustransport signifikant eingeschränkt und wird hauptsächlich durch die Hustenclearance aufrecht erhalten. Auch bei gesunden Probanden verbleibt in Abhängigkeit der Größe der Teilchen eine bestimmte Fraktion über einen längeren Zeitraum ( $>48$ Stunden) in der Lunge $[29,37]$. Die zugrunde liegenden Mechanismen dieser so genannten retardierten Clearance sind noch unklar, bestimmte Partikeleigenschaften, wie Größe und Oberflächenbeschaffenheit scheinen diesen Prozess zu beeinflussen. Den in den Atemwegen angesiedelten Atemwegsmakrophagen kommt beim Verständnis der Mechanismen der retardierten Clearance eine entscheidende Bedeutung zu, so dass auch im Bronchialtrakt mit einem im Vergleich zur Peripherie vergleichbaren makrophagenassoziierten Langzeitreinigungsprozess gerechnet werden muss.

\section{Clearancemechanismen in der Lungenperipherie}

Zur Untersuchung der langsamen alveolaren Clearanceprozesse müssen Radionuklide mit Halbwertszeiten zwischen 3-6 Monaten eingesetzt werden. Wegen der damit verbundenen erhöhten Strahlenexposition gibt es deshalb vor allem tierexperimentelle Studien, aber nur wenige Studien am Menschen [2,26,27]. Als Radionuklide wurden in diesen Studien ${ }^{51} \mathrm{Cr}$, sowie ${ }^{195} \mathrm{Au}$ eingesetzt, die Halbwertzeiten von ca. 6 Monaten haben. Die radioaktive Tracermethode bietet aber für kinetische Untersuchungen den Vorteil, nicht nur die Abnahme der retinierten Testpartikeln im jeweiligen Organ zu untersuchen, sondern auch die Eliminationspfade zu erfassen. Partikeln, die über die mukoziliare „Roll- 
treppe“ aus der Lunge heraustransportiert werden, gelangen in den Magen-Darm-Trakt und können über die Stuhlausscheidung erfasst werden. Die in den Makrophagen gelösten Fraktionen gelangen zunächst in das Blut und können über die Urinausscheidung erfasst werden, falls der Stoff nicht im Körper anderweitig metabolisiert wird. Die wesentlichen Mechanismen der Clearance von Partikeln aus der Peripherie sind intrazellularer Abbau mit der Absorption ins Blut sowie mechanischer Transport und wurden im Abschnitt über das ICRP-Modell erläutert. Voraussetzung für diese, durch die Alveolarmakrophagen induzierte Clearance, ist allerdings die Phagozytose der Partikeln. Den Neutrophilen kommt wegen der kurzen Lebensdauer und dem geringen Anteil bei gesunden Probanden nur eine untergeordnete Bedeutung bei den Clearancemechanismen zu. Bei Patienten mit chronischen Lungenerkrankungen (z. B. chronische Bronchitis) ist die Neutrophilenzahl jedoch stark erhöht, so dass diese auch eine stärkere Bedeutung bei den Clearancemechanismen erlangen.

\section{Phagozytose}

Nach der Ablagerung von Partikeln in der Lungenperipherie kommt es zu einer chemotaktischen Reaktionskaskade, die zu einer Migration der in den Alveolen angesiedelten Makrophagen zum Ort der Partikeldeposition führt. Dem die Alveolen auskleidenden Surfactant kommt dabei eine besondere Bedeutung zu [11]. Durch die Benetzung mit Surfactant wird eine Opsonierung der Partikeln erreicht und eine schnellere Phagozytose durch die Aktivierung von spezifischen Rezeptoren (Komplement) auf den Makrophagen ermöglicht [13]. In-vivo-Untersuchungen der Phagozytose von inhalierten magnetischen Testpartikeln haben Halbwertszeiten von 1-2 Stunden für Lungengesunde ergeben [4]. Dieser Prozess ist bei chronischen Lungenkrankheiten signifikant gestört [7]. Durch den Prozess der Phagozytose werden die Partikeln und Fremdstoffe sehr schnell vom Lungenepithel entfernt, so dass im physiologischen Fall ein steriler Zustand der Lunge gewährleistet wird.

\section{Intrazellulärer Abbau}

Die phagozytierten Partikel müssen von den Makrophagen abgebaut werden. Dazu stehen verschiedene Strategien zur Verfügung. Zunächst kommt es zur Fusion der Phagosomen mit Lysosomen, die eine Reihe von Enzymen enthalten, um Bakterien und Viren abzubauen bzw. zu deaktivieren. Zusätzlich setzt, gesteuert durch Membranrezeptoren, die Produktion von reaktiven Sauerstoffspezies $\left(\mathrm{O}_{2}, \mathrm{H}_{2} \mathrm{O}_{2}\right)$ ein, die Bakterien und Viren deaktivieren. Schwer lösliche anorganische Partikel widerstehen jedoch diesen Abbauprozessen und können über einen längeren Zeitraum in der Lunge verbleiben. Beim Abbau (Lösung) dieser kompakten Partikel innerhalb der Makrophagen spielt das saure Milieu in den Phagolysosomen $(\mathrm{pH} \approx 5)$ zusätzlich eine entscheidende Rolle [25]. Die chemische Lösung von kompakten Partikeln konnte in vitro mit lavagierten Alveolarmakrophagen simuliert werden, jedoch nicht außerhalb von Makrophagen in, den Körperflüssigkeiten vergleichbaren, Lösungen [20]. Die Lösungskomponente der Clearance von Partikeln in der Lunge ist stark abhängig vom inhalierten Material und von dessen Eigenschaften wie Größe, spezifischer Oberfläche und chemischer Zusammensetzung. Die Abbauprodukte (Ionen) gelangen aufgrund von Diffusionsgradienten in die Zirkulation und können dann mit dem Urin ausgeschieden werden, wenn keine anderweitige Metabolisierung des Stoffes im Körper stattfindet. Diese Metabolisierung kann schon in den Makrophagen durch die Kopplung an bestimmte Proteine geschehen, wodurch Transport und Akkumulation in andere Organe induziert werden können. Daraus wird deutlich, dass durch die alleinige Analyse der Ausscheidepfade die Clearance der Partikel aus der Lunge falsch beurteilt werden kann.

\section{Mechanische Clearance}

Bei der Elimination der Partikeln aus der Lunge kommt neben der chemischen Lösung den mechanischen Reinigungspfaden eine zentrale Bedeutung zu: Ein Teil der Makrophagen wandert in Richtung der kleinen Atemwege, wo sie das mukoziliare Schleimtransportsystem erreichen können. Diese, mit Partikeln beladenen Makrophagen verlassen dann die Lunge innerhalb von 24 Stunden, werden mit dem Schleim verschluckt und über den Magen-Darm-Trakt ausgeschieden. Partikeln, bzw. Makrophagen, die diesen Transportweg nicht erreichen, verbleiben permanent in der Lunge. Die Aufteilung zwischen mechanischer Reinigung aufgrund des Transports zu den Atemwegen und der intrazellularen Lösung ist von der Spezies abhängig. Bei Nagern sind die Verhältnisse umgekehrt wie bei Hunden bzw. wie beim Menschen. Bei Nagern verläuft das Transportsystem zu den Atemwegen sehr effektiv, so dass der größere Teil der abgelagerten Partikel vergleichsweise schnell aus der Lunge entfernt werden kann [3,21]. Beim Menschen (und auch beim Hund) hat das mechanische Transportsystem dagegen eine untergeordnete Bedeutung. Nur ein geringerer Anteil der Alveolarmakrophagen kann das mukoziliare Reinigungssystem erreichen. Dies konnte in Clearancestudien mit schwer löslichen Teflonpartikeln nachgewiesen werden, die mit einem langlebigen Radionuklid $\left({ }^{195} \mathrm{Au}\right.$, Halbwertsdauer 183 Tage) markiert waren [27]. Durch parallele Messung der Ausscheidung über den Urin und über den Stuhl konnte die Effektivität der beiden Reinigungspfade verifiziert werden unter der Annahme, dass Au nicht anderweitig im Körper metabolisiert wird. Dabei ergab sich, dass zusammen mit den langen Halbwertsdauern der Lungenreinigung von ca. 700 Tagen der radioaktive Tracer fast ausschließlich im Stuhl nachgewiesen wurde. Die inerten Partikel konnten nicht aufgelöst werden und die Reinigungsmessungen charakterisieren demnach vornehmlich die mechanische Reinigungskomponente, die mit der vorab genannten langen Halbwertsdauer beschrieben werden muss. Diese Mechanismen sind im ICRP-Modell über die Deposition und Retention von inerten Partikeln in der Lunge des Menschen berücksichtigt [16].

Innerhalb der Lunge gibt es Umverteilungsvorgänge, so dass die mit Partikeln beladenen Makrophagen, die nicht die mukoziliare „Rolltreppe“ erreichen, nicht permanent auf dem Lungenepithel verbleiben. Hier sind vor allem das Übertreten der Makrophagen (Migration) in das Lymphsystem sowie in das Interstitium zu erwähnen. Schwer lösliche Teilchen, wie etwa Kohlenstoffpartikel, können deshalb über einen langen Zeitraum in der Lunge verbleiben. Dies kann lange andauernde toxische bzw. inflammatorische Reaktionen triggern [39] bzw. bei radioaktiven Aerosolen zu einer lange anhaltenden Strahlenexposition führen.

\section{Clearance ferromagnetischer Eisenoxidpartikel aus der Lungenperipherie}

Für die Langzeituntersuchung der Alveolarclearance am Menschen bietet die magnetische Tracertechnik (Magnetopneumo- 
graphie) eine Alternative zur radioaktiven Tracertechnik [10,35] Dazu werden ferromagnetische Testpartikel inhaliert, deponiert und mit Hilfe eines sensitiven supraleitenden Magnetfeldsensors (SQUID) nachgewiesen. Diese Methodik sowie die Ergebnisse einer klinischen Studie sollen im Folgenden dargestellt werden.

\section{Produktion, Inhalation und Deposition der Eisenoxidtestpartikel}

Sphärische ferromagnetische Testpartikel $\left(\mathrm{Fe}_{3} \mathrm{O}_{4}\right.$, Magnetit [23]) einheitlicher Größe (monodispers) werden unter kontrollierten Bedingungen, wie Atemzugvolumen, Atemfluss und Atempause inhaliert und als Tracer zur Markierung der alveolaren Reinigungsmechanismen in der Lunge deponiert. Die Partikel haben

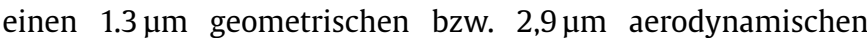
Durchmesser. Partikel mit einem aerodynamischen Durchmesser von $3 \mu \mathrm{m}$ passieren bei gesunden Probanden bei einem Atemfluss von $250 \mathrm{~cm}^{3} / \mathrm{s}$ die Atemwege ohne nennenswerte Ablagerung und dringen bis in die Alveolen vor. Nach einer zusätzlichen Atempause von beispielsweise $4 \mathrm{~s}$ am Ende der Inhalation werden über 70\% der Partikel in der Lunge abgelagert [14]. Die Aerosolsonde (RAP, Respiratory Aerosol Probe [8]), die zur Untersuchung der Lungenstruktur und des konvektiven Gastransports eingesetzt wird, eignet sich besonders gut zur Kontrolle und Überwachung des Inhalationsvorganges. Zur Verminderung der Ablagerung von Partikel in den Atemwegen während der Atempause werden mit Hilfe des Ventilsystems der RAP am Ende des Aerosolbolusses zusätzlich $200 \mathrm{~cm}^{3}$ gefilterte Luft inhaliert. Die Partikeln zeigen eine ausreichende chemische Stabilität und sind in der Lunge über einen Zeitraum von bis zu einem Jahr nachweisbar. Bei der Deponierung von $1 \mathrm{mg}$ der ferromagnetischen Testpartikel ist für die Probanden mit keiner gesundheitlichen Gefährdung zu rechnen [36].

\section{Nachweis der magnetischen Testpartikel mit einem SQUID-System (Magnetopneumographie)}

Die Partikel werden aufgrund ihres remanenten magnetischen Momentes mit Hilfe eines empfindlichen supraleitenden Magnetfeldsensors (SQUID, Superconducting Quantum Interference Device) außerhalb der Lunge nachgewiesen [35]. Dazu werden die Partikel zunächst in einen 0,1 Tesla starken Magnetfeldpuls magnetisiert und ausgerichtet. Danach kann außerhalb des Thorax eine Magnetfeldstärke von ca. 100 picoTesla (10-12 Tesla) gemessen werden. Der hier verwendete Magnetfeldsensor ist ein Einkanal-SQUID-System mit einem Schleifendurchmesser von $10 \mathrm{~cm}$, der als Gradiometer aufgebaut ist. Dadurch wir ein integrales Magnetfeld der in der Lunge deponierten Partikel erfasst mit einer geringen Ortsauflösung. Die magnetische Nachweistechnik wurde erst nach der Entwicklung der hochsensitiven SQUID-Sensoren möglich. Mit Hilfe dieser Sensoren können sehr schwache Magnetfelder von einigen fTesla nachgewiesen werden, was nicht nur die Untersuchung der magnetischen Kontamination der Lunge erlaubt, sondern auch weiterer biomagnetischer Felder, wie das Magnetoenzephalogramm (MEG), sowie das Magnetokardiogramm (MKG) [1]. Dazu werden heute Vielkanalsysteme (bis zu 120 Kanäle bei MEG-Messungen) eingesetzt, die eine relativ hohe zeitliche Auflösung haben und eine gute Ortslokalisation ermöglichen. Zusätzlich ist für das Erreichen einer entsprechend hohen Empfindlichkeit eine effektive Abschirmung äußerer Störfelder notwendig. Die ersten biomagnetischen Messungen zur magnetischen Kontamination der Lunge wurden von Cohen [10] und von Kalliomäki [17] durchgeführt.

\section{Ferromagnetische Kontamination der Lunge}

Bei allen Probanden kann eine geringe ferromagnetische Kontamination der Lunge gefunden werden, die vor jeder Inhalation mit dem SQUID-System gemessen werden muss und im Mittel mit einer Menge von 0,1-0,2 mg Eisenoxid abgeschätzt werden kann. Dieser Betrag wird nach der Inhalation der ferromagnetischen Testpartikel von allen folgenden Messungen subtrahiert. Bei bestimmten Berufsgruppen, die am Arbeitsplatz erhöhten Konzentrationen an ferromagnetischen Stäuben ausgesetzt sind, wie etwa bei Schweißern, konnten mit der magnetopneumographischen Methode Mengen bis über $1000 \mathrm{mg}$ nachgewiesen werden $[17,24]$.

\section{Alveolarclearance bei gesunden Probanden}

Wie bereits im vorigen Abschnitt dargelegt, werden Untersuchungen der Reinigungsfunktion der Lunge (Atemwege) in der Klinik vornehmlich mit radioaktiv markierten Testaerosolen durchgeführt. Wegen der sehr langsam ablaufenden Reinigungsprozesse in der Peripherie eignet sich diese Methode nicht für Studien am gesunden Menschen. Deshalb gibt es nur wenige Studien zu den peripheren Reinigungsmechanismen der Lunge am Menschen. Die magnetische Tracermethode (Magnetopneumographie, MPG) bietet hier einen Ausweg, erfordert jedoch auch einen hohen spezifischen apparativen Aufwand, der nur bedingt einer klinischen Nutzung außerhalb von Studien zugeführt werden kann.

Messungen der Reinigungsfunktion nach der Inhalation von Magnetstäuben wurden erstmals von Cohen durchgeführt [10]. Diese Untersuchungen beschreiben nur eine geringe Probandenzahl; es konnte dennoch erstmals gezeigt werden, dass Zigarettenraucher eine eingeschränkte periphere Reinigungsfunktion aufweisen. Diese Untersuchungen wurden in neueren Arbeiten auf größere Kollektivgruppen ausgeweitet [22,35]. Da diese Studien neben Daten zum Einfluss des Zigarettenrauchens auch Ergebnisse von Patienten mit peripheren bzw. zentralen Lungenerkrankungen enthalten, sollen die Ergebnisse im Folgenden dargestellt und näher erläutert werden. Die Übersichtsdaten der Teilnehmerkollektive sowie einige der Lungenfunktionsparameter sind in Tab. 1 dargestellt. In diesen Studien konnte bereits bei jungen Rauchern (Alter: 20 - 39 Jahre) mit nur 10 Pack-Years (PY) akkumuliertem Zigarettenrauchkonsum (1 PY entspricht dem Rauchen einer Packung Zigaretten pro Tag über einen Zeitraum

Tab. 1 Daten der gesunden Teilnehmer, der Patienten mit Sarkoidose (SAR), mit interstitieller Lungenfibrose (IPF), sowie mit chronischer Bronchitis (COB), jeweils aufgeteilt nach Nichtrauchern (NR), Rauchern (R) und Exrauchern (XR). Signifikanzniveau im Vergleich zu gesunden Probanden: ns: nicht signifikant, ${ }^{*}$ : $p<0,05,{ }^{* *}$ : $p<0,01$

\begin{tabular}{lllll}
\hline & gesund & SAR & IPF & COB \\
\hline Alter (Jahre) & $54 \pm 7$ & $48 \pm 14$ & $49 \pm 15$ & $60 \pm 8$ \\
\hline NR/R/XR & $9 / 8 / 0$ & $10 / 2 / 3$ & $5 / 4 / 3$ & $1 / 3 / 14$ \\
\hline Pack-years (PY) & $49 \pm 18$ & $18 \pm 13$ & $15 \pm 6$ & $40 \pm 25$ \\
\hline FEV $_{1}$ (\%pred.) & $105 \pm 15$ & $93 \pm 15\left({ }^{*}\right)$ & $82 \pm 23\left({ }^{*}\right)$ & $72 \pm 28\left({ }^{* *}\right)$ \\
\hline FEV $/$ /VC (\%pred.) & $91 \pm 10$ & $94 \pm 6(\mathrm{~ns})$ & $94 \pm 15(\mathrm{~ns})$ & $69 \pm 19\left({ }^{* *}\right)$ \\
\hline RV\% TLC & $32 \pm 6$ & $33 \pm 6(\mathrm{~ns})$ & $37 \pm 12(\mathrm{~ns})$ & $45 \pm 10\left({ }^{* *}\right)$ \\
\hline VC (\% pred.) & $118 \pm 13$ & $102 \pm 17\left({ }^{* *}\right)$ & $86 \pm 19\left({ }^{* *}\right)$ & $103 \pm 25(\mathrm{~ns})$ \\
\hline
\end{tabular}


von einem Jahr) eine signifikante Verlängerung der alveolaren Clearancehalbwertsdauer festgestellt werden. Die mittlere Halbwertsdauer der Reinigung erhöhte sich von $124 \pm 66$ Tage (Mittelwerte \pm Standardabweichung, Abb. 2) bei jüngeren Nichtrauchern auf $220 \pm 78$ Tage bei gleichaltrigen Rauchern. Bei älteren Rauchern (Alter: 40-65 Jahre) mit 49 \pm 18 PY erhöhte sich die Reinigungshalbwertsdauer von $162 \pm 120$ Tage auf $459 \pm 334$ Tage. Alle Daten zusammen genommen ergeben eine Verlangsamung der Halbwertsdauer der alveolaren Reinigungsfunktion von $\approx 6$ Tagen pro PY (Abb.3). Diese Untersuchungen belegen klar den toxischen Einfluss das Zigarettenrauchens auf die Funktionen der Lunge. Das zellulare Abwehrsystem ist massiv in seiner Funktion beeinträchtigt. Nach Nikotinkarenz kommt es zu einer teilweisen Erholung der Reinigungskapazität der Lunge [6], die auch bei Patienten mit COB nachgewiesen werden konnten, die überwiegend Exraucher waren und deren Clearancehalbwertsdauer sich trotz $40 \pm 25$ PY verkürzte [22].

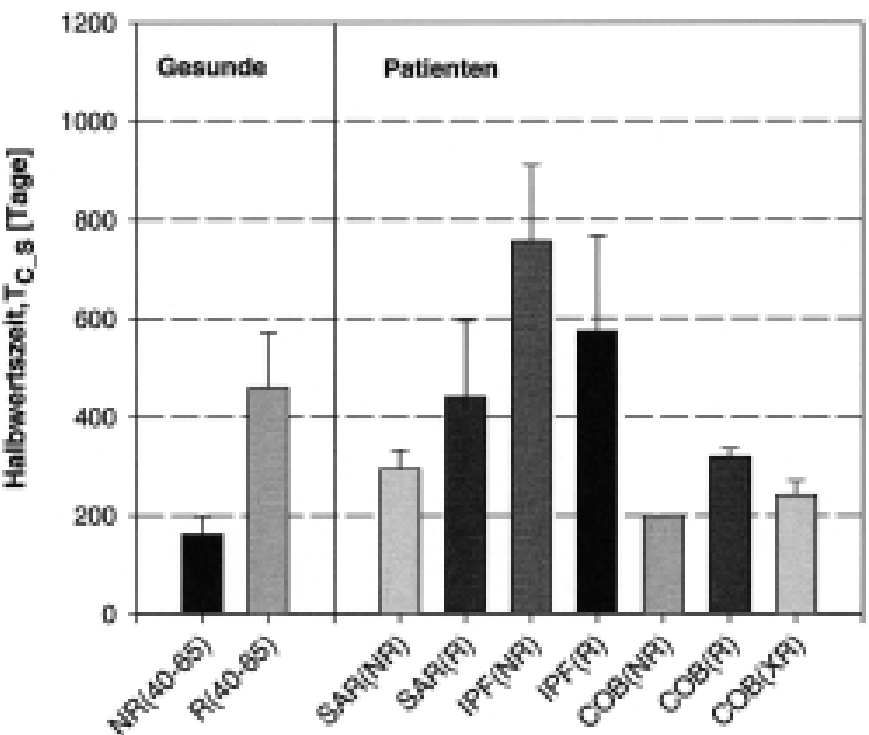

Abb. 2 Clearancehalbwertsdauer ( $T_{C}$, Mittelwerte \pm Standardfehler) bei gesunden Nichtrauchern (NR) und Rauchern (R) aus der Altersgruppe 40-65 Jahre und bei Patienten mit chronisch obstruktiver Bronchitis (COB), interstitieller Lungenfibrose (IPF) sowie mit Sarkoidose (SAR), jeweils unterteilt nach Raucheranamnese [22].

Die Mechanismen der Einschränkung der Makrophagenfunktion durch Zigarettenrauchen sind nicht vollständig geklärt. Da die Clearancestudien am Menschen vor allem die intraphagosomale Lösungskapazität widerspiegeln und damit abhängig sind vom eingesetzten Tracermaterial (hier Eisenoxid), deuten die Clearanceeinschränkungen bei den Rauchern auf veränderte ionische Bedingungen in den Phagolysosomen der Makrophagen hin. Beim Zigarettenrauchen wird eine Vielzahl von chemischen Substanzen in der Lunge abgelagert, die Lösungsmechanismen in den Makrophagen neutralisieren können. Die Daten korrelieren auch mit einer verminderten Antibakterizität der durch bronchoalveolare Lavage (BAL) gewonnenen Alveolarmakrophagen von Rauchern [12,31]. Die Einschränkungen der Abwehrfunktionen im Alveolarbereich durch das Zigarettenrauchen muss auch im Zusammenhang mit einem stark erhöhten Lungenkrebsrisiko gesehen werden, welches sich nach Nikotinkarenz wieder vermindert [30].

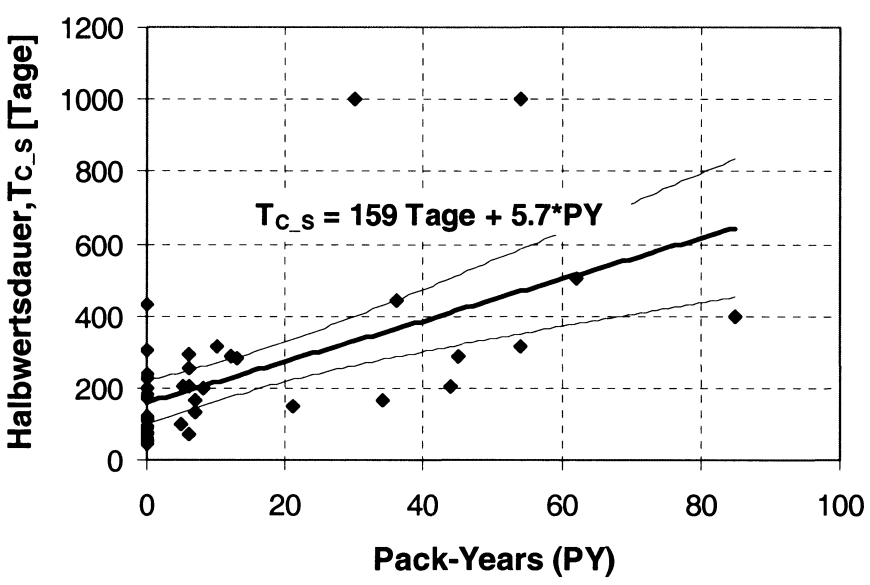

Abb. 3 Korrelation zwischen der langsamen Clearancehalbwertsdauer $\left(T_{C_{-} S}\right)$ und dem kumulierten Zigarettenrauchkonsum (PY) bei den gesunden Probanden $(p<0,01)$ [22].

\section{Alveolarclearance bei Patienten mit Lungenkrankheiten}

Makrophagenfehlfunktionen können eine zentrale Rolle bei der Pathogenese von interstitiellen Lungenkrankheiten spielen. Deshalb wurde die alveolare Reinigungsfunktion bei Patienten mit Sarkoidose (SAR) sowie mit interstitieller Lungenfibrose (IPF) untersucht. Zusätzlich wurden Patienten mit chronisch obstruktiver Bronchitis (COB) in die Studie aufgenommen, um den Einfluss einer primär in den Atemwegen ablaufenden Inflammation auf die Abwehrkapazität der Alveolarmakrophagen mitzuerfassen [22]. Wegen des signifikanten Einflusses des Zigarettenrauchens bei Gesunden müssen die Daten aufgrund dieses Einflussfaktors eingeordnet und bewertet werden. Da COB vornehmlich mit langandauerndem Zigarettenkonsum verknüpft ist, ist von besonderem Interesse, dass die meisten COB-Patienten einen den asymptomatischen Rauchern vergleichbaren Zigarettenkonsumindex (PY) aufweisen, aber oft schon seit mehreren Jahren nicht mehr aktiv rauchen. Diese Patienten wurden in eine eigene Gruppe (COB-Exraucher) eingeordnet. Die Reinigungsfunktion der COB-Exraucher verläuft mit $240 \pm 74$ Tagen Halbwertsdauer deutlich langsamer im Vergleich zu den gesunden Nichtrauchern (162 \pm 120 Tage Halbwertsdauer). Sie hat sich allerdings gegenüber den asymptomatischen Rauchern (mit gleicher Zahl an PY) beschleunigt. Dies belegt, dass sich die alveolare Reinigungsfunktion nach Nikotinkarenz teilweise wieder erholen kann. Bei COB-Patienten wurde innerhalb der ersten 24 Stunden nach Inhalation 50\% der deponierten Magnetpartikeln aus der Lunge heraustransportiert. Es muss also eine hohe Ablagerung der Partikel in den Atemwegen stattgefunden haben, die durch die bei COB vorhandenen bronchialen Obstruktionen (Tab.1) induziert wird. Dies zeigt auch die signifikante Korrelation der Fraktion der schnell gereinigten Partikel mit der forcierten expiratorischen Kapazität $\left(\mathrm{FEV}_{1}\right)$. Bei den gewählten Parametern für Teilchengröße und Inhalationsfluss ist die Fraktion der schnell gereinigten Partikel ein empfindliches Maß für eine Atemwegsobstruktion. Dieser Parameter ist auch bei asymptomatischen Rauchern tendenziell erhöht und zeigt frühe toxische Einflüsse des Zigarettenrauchs auf den Bronchialtrakt an.

Bei den SAR-Patienten (10 Nichtraucher, 2 Raucher, 3 Exraucher) spielte das Rauchen nur eine untergeordnete Rolle. Die alveolare Reinigungsfunktion verläuft gegenüber den nichtrauchenden 
Kontrollprobanden mit $275 \pm 109$ Tagen deutlich langsamer. Es muss also bei SAR trotz aktivierter Makrophagen von einer eingeschränkten Makrophagenabwehrfunktion ausgegangen werden.

Das Krankheitsbild der IPF ist mit einer drastischen Einschränkung der alveolaren Reinigungsfunktion verknüpft. Die Halbwertdauer beträgt auch bei den nichtrauchenden IPF-Patienten $756 \pm 347$ Tage (gesunde Nichtraucher $162 \pm 120$ Tage), wobei bei einer Reihe der IPF-Patienten während des Untersuchungszeitraumes von einem Jahr überhaupt keine Abnahme des magnetischen Tracers zu verzeichnen war. Bei IPF muss demnach von essentiellen Fehlfunktionen der AM ausgegangen werden, die sich auch in der verlangsamten Phagozytosetätigkeit widerspiegelte [4]. Die verminderte Abwehrfunktion bei IPF korreliert ebenso wie bei den asymptomatischen Rauchern mit einem erhöhten Lungenkrebsrisiko [15]. Die beschleunigte DTPA-Clearance [28] bei IPF-Patienten zeigt zusätzlich Schäden an der transepithelialen Luft-Blutschranke an.

\section{Schlussfolgerung}

Die alveolare Clearance schwer löslicher Partikeln verläuft sehr langsam und ist primär an die Funktion der Alveolarmakrophagen geknüpft. Der Transport der Partikel zum Bronchialsystem ist beim Menschen von untergeordneter Bedeutung, so dass die Elimination der Partikel vornehmlich durch die Lösung innerhalb der Makrophagen bestimmt wird. Die magnetische Tracertechnik (Magnetopneumographie) stellt eine nicht-invasive Methode dar, um die langsam ablaufenden alveolaren Reinigungsmechanismen am Menschen zu untersuchen. Lange andauernder Zigarettenrauchkonsum führt zu einer signifikanten Verminderung der Reinigungskapazität der Lunge. Eine Einschränkung der alveolaren Clearance wird auch bei interstitiellen Lungenerkrankungen, wie der Sarkoidose und der interstitiellen Lungenfibrose beobachtet. Das Krankheitsbild der chronischen Bronchitis mit Infekten vor allem in den konduktiven Atemwegen hat nur gerinfügige Auswirkungen auf die alveolare Clearance. Eine eingeschränkte Alveolarclearance kann auch mit einem erhöhten Lungenkrebsrisiko verbunden sein. In zukünftigen Studien müssen die Mechanismen der eingeschränkten Makrophagenfunktion in vitro untersucht werden.

\section{Literatur}

${ }^{1}$ Aine CJ, Okada Y, Stroink G, Swithenby SJ, Wood CC. Biomag 96: Proceedings of the Tenth International Conference on Biomagnetism. New: York: Springer, 2000

2 Bailey MR, Fry FA, James AC. The long-term clearance kinetics of insoluble particles from the human lung. Ann Occup Hyg 1982; 26: $273-290$

${ }^{3}$ Bailey MR, Kreyling WG, Andre S, Batchelor A, Collier CG. An interspecies comparison of the lung clearance of inhaled monodisperse cobalt oxide particles, part I: objectives and summary of results. J Aerosol Sci 1989; 20: 169- 188

${ }^{4}$ Barth W, Möller W, Pohlit W, Stahlhofen W, Wiegand J. Magnetopneumographic estimation of particle phagocytosis in the human lungs. J Aerosol Sci 1994; 25: $491-492$

${ }^{5}$ Bennett WD, Scheuch G, Zeman KL, Brown JS, Kim C, Heyder J, Stahlhofen W. Bronchial airway deposition and retention of particles in inhaled boluses: effect of anatomic dead space. J Appl Physiol 1998; 85: $685-694$
${ }^{6}$ Bohning DE, Atkins HL, Cohn SH. Long-term particle clearance in man: normal and impaired. Ann Occup Hyg 1982; 26: 1 - 4

${ }^{7}$ Brain JD. Mechanisms, measurement, and significance of lung macrophage function. Environ Health Perspect 1992; 97: 5-10

8 Brand P, Häussinger K, Meyer T, Scheuch G, Schulz H, Selzer T, Heyder $\mathrm{J}$. Intrapulmonary distribution of deposited particles. J Aerosol Med 1999; 12: 275-284

${ }^{9}$ Camner P, Anderson M, Philipson K, Bailey A, Hashish A, Jarvis N, Bailey $M$, Svartengren M. Human bronchiolar desposition and retention of 6-, 8- and 10-micrograms particles. Exp Lung Res 1997; 23: $517-535$

${ }^{10}$ Cohen D, Arai SF, Brain JD. Smoking impairs long-term dust clearance from the lung. Science 1979; 204: 514-517

${ }^{11}$ Geiser M, Schuepbach R, Waber U, Gehr P. Bioassay for hamster macrophage chemotaxis: application to study particle-lung interactions. Cell Mol Life Sci 1998; 54: 179-185

${ }^{12}$ Green GM. Mechanisms of tobacco smoke toxicity on pulmonary macrophage cells. Eur J Respir Dis 1985; 139: 82 -85

${ }^{13}$ Hamacher J. Schalberg T. Adhesion moleculares in lung desease. Lung 1994; 172: 189-213

${ }^{14}$ Heyder J, Gebhart J, Rudolf G, Schiller CF, Stahlhofen W. Deposition of particles in the human respiratory tract in the size range $0.005-15$ microns. J Aerosol Sci 1986; 17: $811-825$

${ }^{15}$ Hubbard R, Venn A, Lewis S, Britton J. Lung cancer and cryptogenic fibrosing alveolitis. A population-based cohort study. Am J Respir Crit Care Med 2000; 161: 5-8

${ }^{16}$ ICRP. Human respiratory tract model for radiological protection. A report of a Task Group of the International Commission on Radiological Protection. Ann ICRP 1994; 24: 1- 482

${ }^{17}$ Kalliomäki K, Kalliomäki PL, Kelhae V, Vaaranen V. Instrumentation for measuring the magnetic lung contamination of steel welders. Ann Occup Hyg 1980; 23: 175-184

${ }^{18}$ Kohlhäufl M, Brand P, Häussinger K, Scheuch G, Schulz H, Heyder J. Pneumologische Diagnostik mit Modellaerosolen I. Teil: Intrapulmonale Morphometrie. Grundlagen und erste klinische Forschungsergebnisse. Pneumologie 1997; 51: 40-46

19 Kohlhäufl M, Brand P, Scheuch G, Schulz H, Häussinger K, Heyder J. Pneumologische Diagnostik mit Modellaerosolen. II. Teil: Aerosolbolusdispersion, ein Marker für den konvektiven Gastransport. Grundlagen und erste klinische Forschungsergebnisse. Pneumologie 1999; 53 : $50-56$

${ }^{20}$ Kreyling WG, Godleski JJ, Kariya ST, Rose RM, Brain JD. In vitro dissolution of uniform cobalt oxide particles by human and canine alveolar macrophages. Am J Respir Cell Mol Biol 1990; 2: 413-422

${ }^{21}$ Kreyling WG, Scheuch G. Clearance of particles deposited in the lungs. In: Gehr P, Heyder J. eds. Particle-Lung Interactions. New York, Basel: Marcel Dekker Inc., 1999: 323-376

${ }^{22}$ Möller W, Barth W, Kohlhäufl M, Häussinger K, Stahlhofen W, Heyder J. Human alveolar long-term clearance of ferromagnetic iron-oxide microparticles in healthy and diseased subjects. Exp Lung Res 2001; 27: $547-568$

${ }^{23}$ Möller W, Scheuch G, Sommerer K, Heyder J. Preparation of spherical monodisperse ferrimagnetic iron-oxide microparticles between 1 and $5 \mu \mathrm{m}$ diameter. J Magn Magn Mater 2001; 225: 8-16

${ }^{24}$ Möller W, Stahlhofen W. Magnetic material in the lungs of dental technicians. J Aerosol Sci 1989; 20: 1345-1348

${ }^{25}$ Nilsen A, Nyberg K, Camner P. Intraphagosomal pH in alveolar macrophages after phagocytosis in vivo and in vitro of fluorescein-labeled yeast particles. Exp Lung Res 1988; 14: 197-207

${ }^{26}$ Philipson K, Falk R, Camner P. Long-term lung clearance in humans studies with Teflon particles labeled with chromium-51. Exp Lung Res 1985; 9: $31-42$

27 Philipson K, Falk R, Gustafsson J, Camner P. Long-term lung clearance of 195Au-labeled teflon particles in humans. Exp Lung Res 1996; 22: $65-83$

${ }^{28}$ Rinderknecht J, Shapiro L, Krauthammer M, Taplin G, Wassermann K, Uszler JM, Effros RM. Accelerated clearance of small soluted from the lungs in interstitial lung disease. Am Rev Respir Dis 1980; 121: $105-117$

${ }^{29}$ Scheuch G, Kohlhäufl M, Sommerer K, Lichte H, Hess W, Schulz H, Häussinger K, Heyder J. Pneumologische Diagnostik mit Modellaerosolen, III. Teil: Clearance von Aerosolpartikeln aus den luftleitenden Atemwegen. Pneumologie 1999; 53: 329-336

30 Simonato L, Agudo A, Ahrens W, Benhamou E, Benhamou S, Boffetta P, Brennan P, Darby SC, Forastiere F, Fortes C, Gaborieau V, Gerken M, Gonzales CA, Jockel KH, Kreuzer M, Merletti F, Nyberg F, Pershagen G, 
Pohlabeln H, Rosch F, Whitley E, Wichmann HE, Zambon P. Lung cancer and cigarette smoking in Europe: an update of risk estimates and an assessment of inter-country heterogeneity. Int J Cancer 2001; 91: $876-887$

${ }^{31}$ Sopori ML, Cherian S, Chilukuri R, Shopp GM. Cigarette smoke causes inhibitation of the immune response to intratracheally administered antigens. Toxical Appl Pharmacol 1989; 97: 489-499

32 Stahlhofen W, Gebhart J, Heyder J, Philipson K, Camner P. Intercomparison of regional deposition of aerosol particles in the human respiratory tract and their long-term elimination. Exp Lung Res 1981; 2: $131-139$

${ }^{33}$ Stahlhofen W, Gebhart J, Rudolf G, Scheuch G. Measurement of lung clearance with pulses of radioactively-labelled aerosols. J Aerosol Sci 1986; 17: 333-336

${ }^{34}$ Stahlhofen W, Köbrich R, Rudolf G, Scheuch G. Short-term and longterm clearance of particles from the upper human respiratory tract as function of particle size. J Aerosol Sci 1990; 21: 407-410

${ }^{35}$ Stahlhofen W, Möller W. Behaviour of magnetic micro-particles in the human lung. Radiat Environ Biophys 1993; 32: 221 - 238

${ }^{36}$ Stokinger HE. A review of world literature finds iron oxides noncarcinogenic. Am Ind Hyg Assoc J 1984; 45: 127 - 133

37 Svartengren M, Sommerer K, Scheuch G, Kohlhäufl M, Heyder J, Falk R, Bergmann R, Hofmann W, Bailey M, Philipson K, Camner P. Comparison of clearance of particle inhaled with bolus and extremely slow inhalation techniques. Exp Lung Res 2001; 27: 367-386

38 Vastag E, Matthys H, Köhler D, Gronbeck L, Daikeler G. Mucociliary clearance and airways obstruction in smokers, ex-smokers and normal subjects who never smoked. Eur J Respir Dis Suppl 1985; 139: $93-100$

${ }^{39}$ Warheit DB, Hansen JF, Yuen IS, Kelly DP, Snajdr SI, Hartsky MA. Inhalation of high concentration of low toxicity dusts in rats results in impaired pulmonary clearance mechanisms and persistent inflammation. Toxicol Appl Pharmacol 1997; 145: 10-22

${ }^{40}$ Weibel ER. Morphometry of the human lung. Berlin, Göttingen, Heidelberg: Springer Verlag, 1963

\section{Preisverleihungen}

im Rahmen des 43. Kongresses

der Deutschen Gesellschaft für Pneumologie

Die Preisträger des Doktorandenpreises

der Deutschen Lungenstiftung

(gestiftet von der Firma Boehringer Ingelheim Pharma KG)

\section{Erster Preis für die beste klinische Arbeit:}

Frau Dr. Jutta Beier, Mainz

Titel: Methodische und klinische Aspekte der Bestimmung von Glutathion im induzierten Sputum bei bronchopulmonalen Erkrankungen

Glutathion ist ein wichtiger Marker für den oxidativen Stress und spielt bei chronisch entzündlichen Bronchial- und Lungenparenchymerkrankungen eine große Rolle. Bislang ließ sich diese Substanz mit ausreichender Zuverlässigkeit nur in der bronchoalveolären Lavage bestimmen. Frau Dr. Beier hat nachgewiesen, dass die Messung dieser wichtigen Substanz auch im so genanten induzierten Sputum möglich ist. Sie hat die Einflussfaktoren sehr genau untersucht, die das Messergebnis verfälschen könnten. Erste Ergebnisse haben die Vergleichbarkeit mit der Glutathionbestimmung in der bronchoalveolären Lavage bestätigt. Es handelt sich um einen wichtigen klinischen Beitrag zur besseren Bestimmbarkeit von chronisch entzündlichen Lungen- und Bronchialprozessen.

\section{Erster Preis für die beste experimentelle Arbeit: Herr Dr. David Groneberg, Berlin}

Titel: Expression, Lokalisation und funktionelle Aspekte des Peptidtransporters PEPT2 im gesunden Atemtrakt und bei Mukoviszidose

Peptide sind wichtige Mediatoren der Lunge. Ihre Aktivität wird durch Peptidasen determiniert. Sie entsteht beim Abbau von Proteinen und mikrobiellen Stoffen und verursachen eine deutliche Störung des Gasaustausches. Dem Abtransport von Peptidfragmenten kommt eine wichtige Bedeutung zu.

Herr Dr. Groneberg hat den genannten Peptidtransporter in der Lunge (Pneumozyten Typ II, Epithel- und Endothelzellen der kleinen Bronchien) bei der Maus und beim Menschen nachgewiesen. Das Interessante ist, dass dieser Peptidtransporter auch so genannte Peptidpharmaka, z.B. bestimmte Antibiotika, transportiert. Insofern könnte dieser Peptidtransporter als ein wichtiges Vehikel für auch inhalativ zu gebende Antibiotika eine große Rolle spielen, was z.B. für die Therapie der Mukoviszidose von großer Bedeutung sein könnte. 